\title{
Complex Domain Wavelet-Based Denoising of Measured UHF Wireless Channel Power Delay Profiles
}

\author{
Maurício Henrique Costa Dias ${ }^{1}$, Gláucio Lima Siqueira ${ }^{2}$ \\ ${ }^{1}$ Department of Electrical Engineering, Military Institute of Engineering, Rio de Janeiro, Brazil \\ ${ }^{2}$ Center for Telecommunications Studies, Pontifical Catholic University of Rio de Janeiro, Rio de Janeiro, Brazil \\ Email:mhcdias@ime.eb.br,glaucio@cetuc.puc-rio.br
}

Received December 29, 2009; revised January 31, 2010; accepted February 25, 2010

\begin{abstract}
This work extends the use of wavelet-based denoising as an alternative processing scheme to improve measured mobile-radio channel power delay profiles. It has already been reported that, when applied on real domain data (amplitude only), denoising provides mainly a qualitative improvement. Here, phase content was also considered, leading to significant qualitative and quantitative improvement of the processed profiles. Signal-to-noise ratios and dynamic ranges improvements as high as $50 \mathrm{~dB}$ have been observed.
\end{abstract}

Keywords: Wireless Propagation Channel, Wideband Channel Sounding, UHF Measurements, Wavelets, Denoising

\section{Introduction}

In wireless wideband propagation channel characterization, time dispersion statistical analysis may be carried out on measurements of the channel impulse response. Local ensemble averages of the time-variant channel responses are very useful in that sense, and are commonly referred to as power delay profiles (PDP) [1]. However, noise, interference and sounder setup imperfections are also present as spurious contributions to the actual PDP, distorting its statistical moments, like mean delay and rms delay spread. In order to minimize those effects to the channel statistics, thresholding procedures are usually adopted. The basic approach consists in establishing a noise threshold, below which the response is simply cut off. The threshold may be determined by a visual inspection or may be guided by noise level estimation [1]. Some additional refinements may also be included, as in [2].

Recently, wavelet-based denoising has been proposed as an alternative cleaning processing scheme. Tests on measured PDP from both outdoor and indoor surveys at UHF frequencies have pointed out qualitative improvement, with smoother noise floors, and also small increases on signal-to-noise ratios (SNR) of the profiles [3]. In the present work, wavelet-based denoising is adopted once again to clean delay profiles, this time taking advantage of the phase content of measured impulse responses, available when frequency domain sounding [1] is carried out, to further improve the data processing.

The rest of this text is organized as follows. Section 2 briefly describes the measured channel data processed in this work. The denoising procedure adopted is addressed in Section 3. The next section presents and discusses some results of this processing technique. Section 5 concludes this work.

\section{Available Measured Data Description}

The available data used to test this processing technique have been measured with the frequency domain channel sounder described in [4], depicted in Figure 1. The sounder was basically a vector network analyzer (VNA) with the propagation channel taken as its device under test (DUT). The sounder operated with a $200 \mathrm{MHz}$ band centred on $1.8 \mathrm{GHz}$. Each recorded impulse response was sampled at a $1 / 801$ rate.

The whole survey has been carried out in two different indoor scenarios: one within the corridors of a university building and the other within a little mall, as described in details in [3]. Both line-of-sight and out-of-sight conditions have been tested. The experiment tried to simulate a pedestrian's walk, so the mobile unit had a speed close to $0.3 \mathrm{~m} / \mathrm{s}$. Considering the whole processing time of the sounder, around two impulse responses per second could be stored. In the overall, more than 3500 channel responses have been measured, from 13 different routes. 


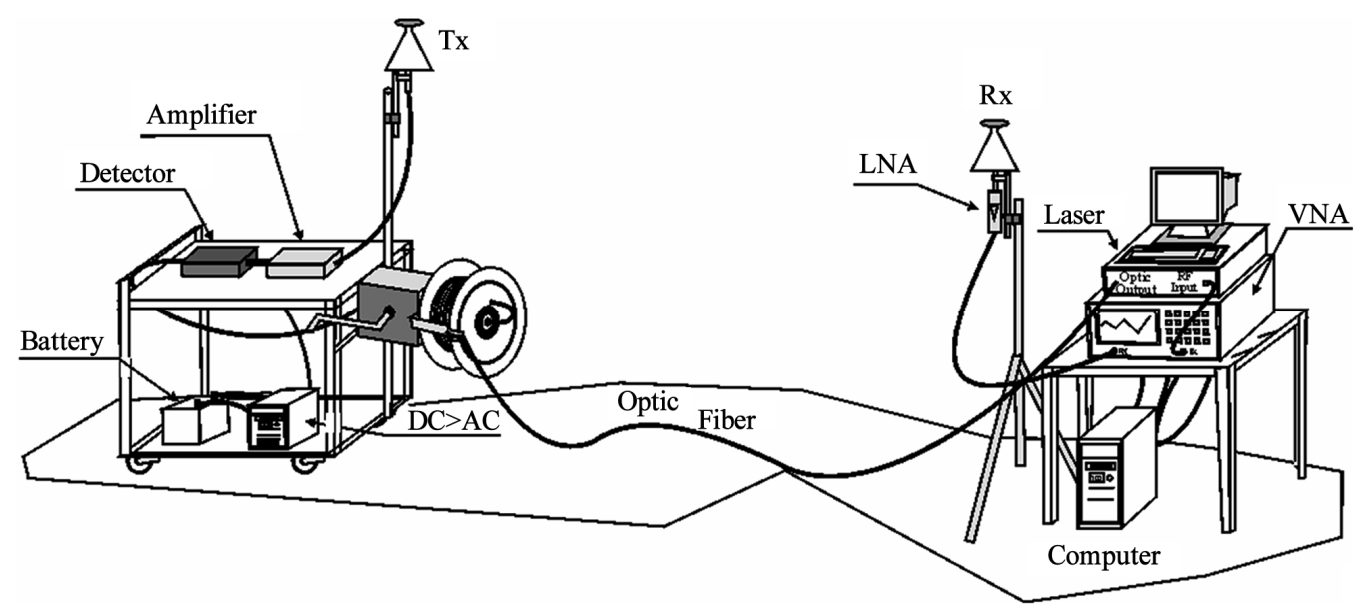

Figure 1. Sounder setup.

The available PDP presented SNR ranging from 21 to 61 $\mathrm{dB}$, and dynamic ranges (DR) within 24 to $61 \mathrm{~dB}$. More information on the survey is found in [3].

\section{Denoising Procedure}

The software tool used to test the proposed technique was Matlab ${ }^{\mathrm{TM}}$, including its wavelet toolbox and functions libraries. Matlab has an automated denoising function based on a dyadic discrete decomposition, with a few user-defined parameters, such as the wavelet function and the number of decomposition levels. Another important user-defined parameter is threshold rescaling as a function of noise level. Actually, two practical options are available: one adopts a white noise assumption, while the other assumes coloured noise.

In [3], the denoising function was applied only on the amplitude of the impulse responses, that is, it was a unidimensional (1D) operator. However, the sounder output was actually complex, rather than real, that is, both amplitude and phase variations of the channel were recorded. In fact, the first approach adopted in [3] disregarded the available relevant phase information. A better approach that tries to benefit from the channel phase content is denoising both real and imaginary projections of the complex channel impulse response (or its counterpart, the power delay profile). Each projection should be independently denoised just like in [3]. Such procedure has been applied to the available indoor data ensemble previously described, and will be referred to as " $2 \mathrm{D}$ denoising” from this point on.

\section{Results}

The trials carried out in [3] provided some insights regarding the best combination of parameters to be chosen for the denoising processing. Since the data ensemble to be $2 \mathrm{D}$ denoised was the same, only the options that led to the best results in [3] have been chosen. In special, the Symlet8 wavelet function has been adopted, as well as Donoho's VisuShrink soft thresholder [5]. 2D denoising has been applied on the delay domain, in the linear scale, and the noise level rescaling method adopted was the one that considered a coloured noise assumption.

As in the 1D approach, 2D denoising has been computed to all available individual channel responses. In order to better assess the results, average power delay profiles have also been generated for each route. Figure 2 illustrates the performance of the 2D denoising procedure on a single channel response. As it can be seen, the noise floor drops more than $40 \mathrm{~dB}$, and the noise oscillations almost fade away.

1D denoising presented mainly qualitative improvements, as already thoroughly discussed in [3]. The

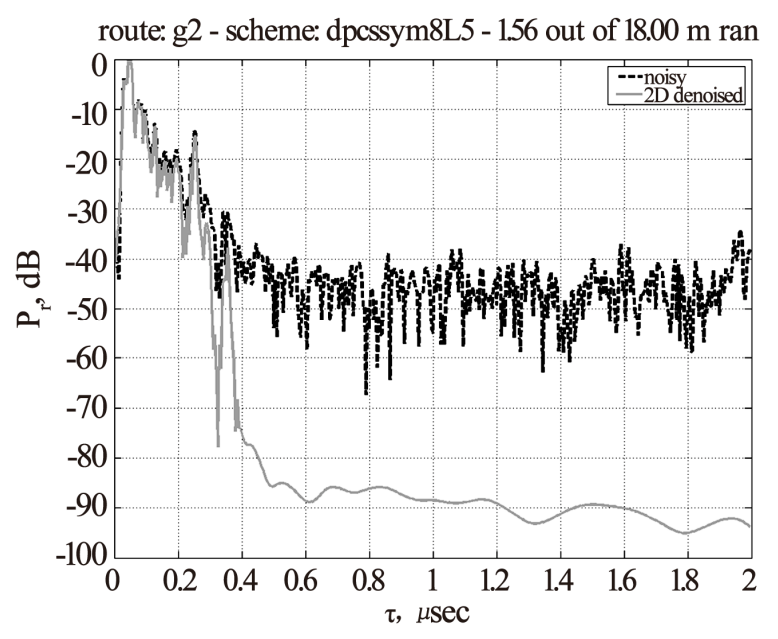

Figure 2. Noisy and 2D denoised channel response sample from $\mathrm{G} 2$ route. 
performance of the 2D denoising was much better, as illustrated in Figures $\mathbf{3}$ and 4, which also present the widely adopted flat thresholding technique [1], just for comparison. Not only was the noise floor flattened, but also significantly lowered, leading to much higher SNR and DR - as high as $50 \mathrm{~dB}$ improvements have been

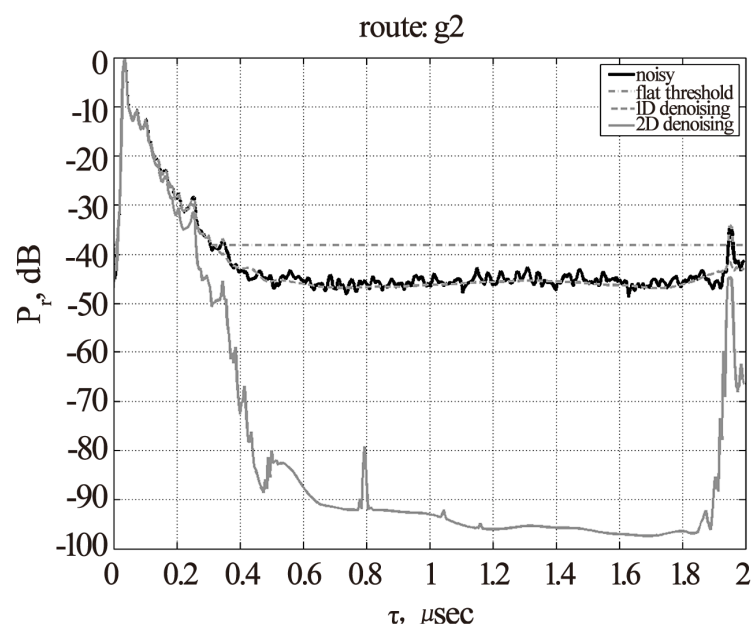

Figure 3. Noisy, denoised (1D and 2D) and flat noise thresholded (10 dB above the mean noise floor) average PDP from $\mathrm{G} 2$ route.

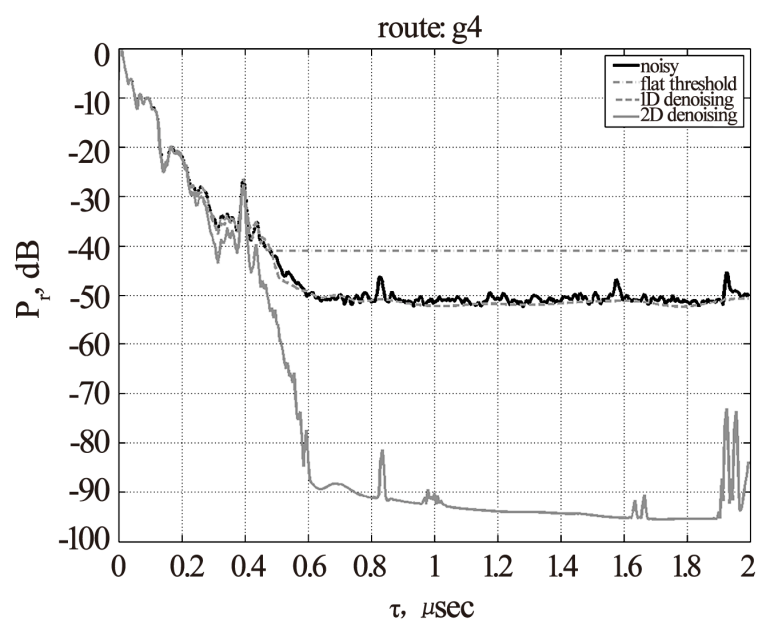

Figure 4. Noisy, denoised (1D and 2D) and flat noise thresholded (10 dB above the mean noise floor) average PDP from $\mathrm{G} 4$ route. achieved, against 3 to $6 \mathrm{~dB}$ provided by 1D denoising. Some potential hidden peaks were even identified, though no theoretical or simulation based procedure has been tested to confirm such peaks as valid ones.

\section{Conclusions}

This work presented an improved version of the wavelet-based denoising scheme proposed in [3] to process wireless channel power delay profiles. While in [3] only the amplitude content of the profiles was processed (1D denoising), in the present work the phase content was also considered (2D denoising).

In order to show how this complementary approach could improve the profiles even more, the same wideband $1.8 \mathrm{GHz}$ indoor measurements described in [3] were considered in the present work. Indeed, while 1D denoising provided mostly a qualitative contribution, with a clear noise floor flattening, 2D denoising led also to significant quantitative improvement. The noise floor was lowered up to $50 \mathrm{~dB}$ in some cases, against no more than $6 \mathrm{~dB}$ when $1 \mathrm{D}$ denoising was adopted.

\section{References}

[1] T. S. Rappapport, "Wireless communications - Principles \& practice,” 2nd Edition, Upper Saddle River, Prentice Hall, 2002.

[2] E. S. Sousa, V. M. Jovanovic, and C. Daigneault, "Delay spread measurements for the digital cellular in Toronto," IEEE Transactions on Vehicular Technology, Vol. 43, No. 4, pp. 837-847, November 1994.

[3] M. H. C. Dias and G. L. Siqueira, "On the use of wavelet-based denoising to improve power delay profile estimates from $1.8 \mathrm{GHz}$ indoor wideband measurements," Wireless Personal Communications, Vol. 32, No. 2, pp. 153-175, January 2005.

[4] L. H. Macedo, M. H. C. Dias, R. D. Vieira, J. F. Macedo, and G. L. Siqueira, "Mobile indoor wide-band $1.8 \mathrm{GHz}$ sounding: measurement-based time dispersion analysis," in Proceedings of the IEEE 55th Vehicular Technology Conference - VTC Spring 2002, Birmingham - AL, USA, Vol. 1, pp. 375-379, May 2002.

[5] D. L. Donoho, “De-noising by soft-thresholding,” IEEE Transactions on Information Theory, Vol. 41, No. 3, pp. 613-627, May 1995. 